\title{
Survival probability of a critical multi-type branching process in random environment
}

\author{
Elena Dyakonova $\|^{p}$ \\ ${ }^{1}$ Department of Discrete Mathematics, Steklov Mathematical Institute, Gubkin St. 8, 119991 Moscow, Russia
}

We study a multi-type branching process in i.i.d. random environment. Assuming that the associated random walk satisfies the Doney-Spitzer condition, we find the asymptotics of the survival probability at time $n$ as $n \rightarrow \infty$.

Keywords: branching processes in random environment, Doney-Spitzer condition, survival probability

\section{Introduction}

Branching processes in random environment constitute an important part of the theory of branching processes (see, for example, (1), (2), (4)-(7), (9)-(14)). A branching process in random environment was first considered by Smith and Wilkinson (10). The subsequent papers (2), (7), (11) investigated single- and multi-type Galton-Watson processes in random environment. The asymptotics of the survival probability of the critical branching processes in a random environment generated by a sequence of independent identically distributed random variables under the condition $\mathbf{E} X^{2}<\infty$ for the increment $X$ of the associated random walk was found in (6), (9) for single-type processes, and in (4) for multi-type processes. Recent papers (1), (5), (12)-(14) study the survival probability for an extended class of the critical single-type branching processes in random environment where the case $\mathbf{E} X^{2}=\infty$ is not excluded and, moreover, $\mathbf{E} X$ may not exist. The present paper investigates an extended class of multi-type critical branching processes in random environment whose associated random walks satisfy the Doney-Spitzer condition. In particular, we generalize some results established in (1) and (4) concerning the asymptotic behavior of survival probability.

Let $Z(n)=\left(Z_{1}(n), \ldots, Z_{p}(n)\right), n=0,1, \ldots$, be a $p$-type Galton-Watson branching process in a random environment. This process can be described as follows.

Let $\mathbf{N}_{0}=\{0,1,2, \ldots\}$ and $\mathbf{N}_{0}^{p}$ be the set of all vectors $t=\left(t_{1}, \ldots, t_{p}\right)$ with non-negative integer coordinates. Denote by $\left(\Delta_{1}, \mathcal{B}\left(\Delta_{1}\right)\right)$ a set of probability measures on $\mathbf{N}_{0}^{p}$ with $\sigma$-algebra $\mathcal{B}\left(\Delta_{1}\right)$ of Borel sets endowed with the metric of total variation, and by $(\Delta, \mathcal{B}(\Delta))$ the $p$-times product of the space $\left(\Delta_{1}, \mathcal{B}\left(\Delta_{1}\right)\right)$ on itself. Let $\mathbf{F}=\left(\mathbf{F}^{(1)}, \ldots, \mathbf{F}^{(p)}\right)$ be a random variable (random measure) taking values in $(\Delta, \mathcal{B}(\Delta))$. An infinite sequence $\Pi=\left(\mathbf{F}_{0}, \mathbf{F}_{1}, \mathbf{F}_{2}, \ldots\right)$ of independent identically distributed copies of $\mathbf{F}$ is said to form a random environment and we will say that $\mathbf{F}$ generates $\Pi$. A sequence of random $p-$ dimensional vectors $Z(0), Z(1), Z(2), \ldots$ with non-negative integer coordinates is called a $p$-type branching process in random environment $\Pi$, if $Z(0)$ is independent of $\Pi$ and for all $n \geq 0, z=\left(z_{1}, \ldots, z_{p}\right) \in \mathbf{N}_{0}^{p}$ and $f_{0}, f_{1}, \ldots \in \Delta$

$$
\begin{aligned}
\mathcal{L}(Z & \left.(n+1) \mid Z(n)=\left(z_{1}, \ldots, z_{p}\right), \Pi=\left(f_{1}, f_{2}, \ldots\right)\right) \\
& =\mathcal{L}\left(Z(n+1) \mid Z(n)=\left(z_{1}, \ldots, z_{p}\right), \mathbf{F}_{n}=f_{n}\right) \\
& =\mathcal{L}\left(\left(\xi_{n, 1}^{(1)}+\cdots+\xi_{n, z_{1}}^{(1)}\right)+\left(\xi_{n, 1}^{(2)}+\cdots+\xi_{n, z_{2}}^{(2)}\right)+\cdots+\left(\xi_{n, 1}^{(p)}+\cdots+\xi_{n, z_{p}}^{(p)}\right)\right),
\end{aligned}
$$

where $f_{n}=\left(f_{n}^{(1)}, f_{n}^{(2)}, \ldots, f_{n}^{(p)}\right) \in \Delta, \xi_{n, 1}^{(i)}, \xi_{n, 2}^{(i)}, \ldots, \xi_{n, z_{i}}^{(i)}, i=1, \ldots, p$, are independent $p$-dimensional random vectors, and for each $i=1, \ldots, p$ the random vectors $\xi_{n, 1}^{(i)}, \xi_{n, 2}^{(i)}, \ldots, \xi_{n, z_{i}}^{(i)}$ are identically distributed according to the measure $f_{n}^{(i)}$. Relation 11 defines a branching Galton-Watson process $Z(n)$ in random environment which describes the evolution of a particle population $Z(n)=\left(Z_{1}(n), \ldots, Z_{p}(n)\right)$, $n=$ $0,1, \ldots$, where $Z_{i}(n), i=1, \ldots, p$, is the number of type $i$ particles in the $n$-th generation.

\footnotetext{
${ }^{\dagger}$ Supported by RFBR grant 05-01-00035, grant Scientific School-4129.2006.1 and by the program "Contemporary Problems of Theoretical Mathematics" of the Russian Academy of Sciences.
} 
This population evolves as follows. If $\mathbf{F}_{n}=f_{n}$ then each of the $Z_{i}(n)$ particles of type $i$ existing at the time $n$, produces offspring in accordance with the $p$-dimensional probability measure $f_{n}^{(i)}$ independently of the reproduction of other particles. Thus, the $i$-th component of the vector $Z(n+1)=\left(Z_{1}(n+1)\right.$, $\left.\ldots, Z_{p}(n+1)\right)$ is equal to the number of type $i$ particles among all direct descendants of the particles of the $n-$ th generation. The distribution of $Z(0)$ will be specified later.

\section{The main results}

Let $J^{p}$ be the set of all column vectors $s=\left(s_{1}, \ldots, s_{p}\right)^{T}, 0 \leq s_{i} \leq 1, i=1, \ldots, p$. For $s \in J^{p}$ and $t \in \mathbf{N}_{0}^{p}$ set $s^{t}=\prod_{i=1}^{p} s_{i}^{t_{i}}$. Taking into account existence of a one-to-one correspondence between probability measures and generating functions we associate with $\mathbf{F}=\left(\mathbf{F}^{(1)}, \ldots, \mathbf{F}^{(p)}\right)$ generating $\Pi$ a random $p$-dimensional column vector $F(s)=\left(F^{(1)}(s), \ldots, F^{(p)}(s)\right)^{T}, s \in J^{p}$, whose components are $p$-dimensional (random) generating functions $F^{(i)}(s)$ corresponding to $\mathbf{F}^{(i)}, 1 \leq i \leq p$ :

$$
F^{(i)}(s)=\sum_{t \in \mathbf{N}_{0}^{p}} \mathbf{F}^{(i)}(\{t\}) s^{t}, s \in J^{p} .
$$

In a similar way we associate with the component $\mathbf{F}_{n}=\left(\mathbf{F}_{n}^{(1)}, \ldots, \mathbf{F}_{n}^{(p)}\right), n \geq 0$, of the random environment $\Pi=\left(\mathbf{F}_{0}, \mathbf{F}_{1}, \mathbf{F}_{2}, \ldots\right)$ a random vector $F_{n}(s)=\left(F_{n}^{(1)}(s), \ldots, F_{n}^{(p)}(s)\right)^{T}, s \in J^{p}$, the components of which are multidimensional (random) generating functions $F_{n}^{(i)}(s)$, corresponding to $\mathbf{F}_{n}^{(i)}, 1 \leq i \leq p$,

$$
F_{n}^{(i)}(s)=\sum_{t \in \mathbf{N}_{0}^{p}} \mathbf{F}_{n}^{(i)}(\{t\}) s^{t} .
$$

Let $e_{j}, j=1, \ldots, p$, be the $p$-dimensional row vector whose $j$-th component is equal to 1 and the others are zeros, $\overline{0}=(0, \ldots, 0)$ be the $p$-dimensional row vector all whose components are zeros, and let $\overline{1}=(1, \ldots, 1)^{T}$ be the $p$-dimensional column vector all whose components are equal to 1 . For $x=\left(x_{1}, \ldots, x_{p}\right)$ and $y=\left(y_{1}, \ldots, y_{p}\right)^{T}$ we set $|x|=\sum_{i=1}^{p}\left|x_{i}\right|,|y|=\sum_{i=1}^{p}\left|y_{i}\right|,(x, y)=\sum_{i=1}^{p} x_{i} y_{i}$. Let $A=\|A(i, j)\|_{i, j=1}^{p}$ be an arbitrary positive $p \times p$ matrix. Denote by $\rho(A)$ the Perron root of $A$ and by $u(A)=\left(u_{1}(A), \ldots, u_{p}(A)\right)^{T}$ and $v(A)=\left(v_{1}(A), \ldots, v_{p}(A)\right)$ the right and left eigenvectors of $A$ corresponding to the eigenvalue $\rho(A)$ and such that

$$
|v(A)|=1,(v(A), u(A))=1 .
$$

For vector-valued generating functions $F(s)$ and $F_{n}(s)$ we introduce the mean matrices

$$
M=M(\mathbf{F})=\|M(i, j)\|_{i, j=1}^{p}=\left\|\frac{\partial F^{(i)}(\overline{1})}{\partial s_{j}}\right\|_{i, j=1}^{p}
$$

and

$$
M_{n}=M_{n}\left(\mathbf{F}_{n}\right)=\left\|M_{n}(i, j)\right\|_{i, j=1}^{p}=\left\|\frac{\partial F_{n}^{(i)}(\overline{1})}{\partial s_{j}}\right\|_{i, j=1}^{p} .
$$

Let $\mathcal{C}_{\alpha}, 0<\alpha<1$, be the class of all matrices $A=\|A(i, j)\|_{i, j=1}^{p}$ such that

$$
\alpha \leq \frac{A\left(i_{1}, j_{1}\right)}{A\left(i_{2}, j_{2}\right)} \leq \alpha^{-1}, 1 \leq i_{1}, i_{2}, j_{1}, j_{2} \leq p .
$$

One of our basic hypotheses is the following condition.

Assumption A0. There exist a number $0<\alpha<1$ and a positive row vector $v=\left(v_{1}, \ldots, v_{p}\right),|v|=1$, such that, with probability 1

$$
M=M(\mathbf{F}) \in \mathcal{C}_{\alpha},
$$

and

$$
v M=\rho(M) v .
$$

Set $\rho=\rho(M), \rho_{n}=\rho\left(M_{n}\right), n \geq 0$. It is not difficult to see that in our settings $X:=\ln \rho, X_{i}:=$ $\ln \rho_{i-1}, i \geq 1$, are independent and identically distributed random variables. Our next hypothesis imposes a restriction on the so-called associated random walk $S=\left(S_{0}, S_{1}, \ldots\right)$, where

$$
S_{n}=X_{1}+\cdots+X_{n}, n \geq 1, S_{0}=0 .
$$


Assumption A1. There exists a number $0<a<1$ such that

$$
\mathbf{P}\left(S_{n}>0\right) \rightarrow a, n \rightarrow \infty
$$

Extending the known classification of single-type branching processes in random environment (see (1), (12)), we call a $p$-type branching process $Z(n), n \geq 0$, in random environment $\Pi$ critical if its associated random walk is of the oscillating type, i.e., $\limsup _{n \rightarrow \infty} S_{n}=+\infty$ a.s. and $\liminf _{n \rightarrow \infty} S_{n}=-\infty$ a.s. It is known that any random walk satisfying Assumption A1 oscillates. From now on we consider only critical $p$-type branching processes in random environment.

Let $0=: \gamma_{0}<\gamma_{1}<\ldots$ be the strict descending ladder epochs of $S$. Put

$$
V(x):=\sum_{i=0}^{\infty} \mathbf{P}\left(S_{\gamma_{i}} \geq-x\right), x \geq 0 ; V(x)=0, x<0 .
$$

Since $S$ is oscillating, the following relation holds (3):

$$
\mathbf{E} V(x+X)=V(x), x \geq 0 .
$$

For $d \in \mathbf{N}_{0}$ set

$$
O_{d}=\left\{t=\left(t_{1}, \ldots, t_{p}\right) \in \mathbf{N}_{0}^{p} \mid t_{i}<d, i=1, \ldots, p\right\}, U_{d}=\mathbf{N}_{0}^{p} \backslash O_{d} .
$$

Introduce the random variable

$$
\kappa(d)=\sum_{t \in U_{d}} \sum_{i=1}^{p} v_{i} \sum_{j, k=1}^{p} \mathbf{F}^{(i)}(\{t\}) t_{j} t_{k} / \rho^{2}, d \in \mathbf{N}_{0},
$$

where $v=\left(v_{1}, \ldots, v_{p}\right)$ is from 2). Our next condition is connected with the random variable $\kappa(d)$, which is a generalization of the standardized truncated second moment of the reproduction law to the multi-type case.

Assumption A2. There exist $\varepsilon>0$ and $d \in \mathbf{N}_{0}$ such that

$$
\mathbf{E}\left(\ln ^{+} \kappa(d)\right)^{1 / a+\varepsilon}<\infty, \mathbf{E}\left(V(X)\left(\ln ^{+} \kappa(d)\right)^{1 / a+\varepsilon}\right)<\infty
$$

Let $T=\min \{n \geq 0: Z(n)=\overline{0}\}$ be the extinction moment for $Z(n)$. Introduce the random variables

$$
Q^{(i)}(n)=\mathbf{P}\left(T>n \mid Z(0)=e_{i}, \Pi\right), Q(n)=\left(Q^{(1)}(n), \ldots, Q^{(p)}(n)\right),
$$

and let

$$
q_{i}(k)=\mathbf{P}\left(T>k \mid Z(0)=e_{i}\right)=\mathbf{E} Q^{(i)}(k) .
$$

Note that under Assumptions A0 and A1 $Q^{(i)}(n) \rightarrow 0 \mathbf{P}-$ a.s. as $n \rightarrow \infty$ for all $1 \leq i \leq p$, since $\mathbf{P}-$ a.s.

$$
(v, Q(n)) \leq \min _{0 \leq k \leq n-1}\left|v M_{0} \cdots M_{k}\right| \leq \exp \left\{\min _{0 \leq k \leq n-1} S_{k}\right\} \rightarrow 0
$$

as $n \rightarrow \infty$. Denote by $u(n)=\left(u_{1}(n), \ldots, u_{p}(n)\right)^{T}:=u\left(M_{0} \cdots M_{n}\right), n \geq 0$, the right eigenvector of the product $M_{0} \cdots M_{n}$, corresponding to the Perron root $\rho\left(M_{0} \cdots M_{n}\right)=\rho_{0} \cdots \rho_{n}$. To investigate the asymptotic behavior of $q_{i}(n)$ and $Q^{(i)}(n)$ as $n \rightarrow \infty$ we need the following statement describing the behavior of $u(n)$.

Theorem 1 If Assumption AO is valid, then there exist a random vector $u=\left(u_{1}, \ldots, u_{p}\right)^{T}$ and a function $g(n) \geq 0, g(n) \rightarrow 0, n \rightarrow \infty$, such that with probability 1

$$
\left|u_{i}(n)-u_{i}\right| \leq g(n), i=1, \ldots, p .
$$

In addition,

$$
(v, u)=1, \alpha \leq u_{i} \leq 1 / v^{*},
$$

where $v^{*}=\min \left(v_{1}, \ldots, v_{p}\right)$ and $v=\left(v_{1}, \ldots, v_{p}\right)$ is from $(2)$.

The following statement describes the behavior of $Q(n)$ as $\mathrm{n} \rightarrow \infty$. 
Theorem 2 Assume Assumptions AO and A1. Then $\mathbf{P}-$ a.s., as $n \rightarrow \infty$,

$$
\frac{Q_{i}(n)}{(v, Q(n))} \rightarrow u_{i}, i=1, \ldots, p,
$$

where $u=\left(u_{1}, \ldots, u_{p}\right)$ is from Theorem 1 .

Now we are ready to formulate the main result of the paper.

Theorem 3 Assume Assumptions A0, A1, and A2. Then, as $n \rightarrow \infty$,

$$
q_{i}(n) \sim c_{i} n^{-(1-a)} l(n), c_{i}>0, i=1, \ldots, p,
$$

where $l(n)$ is a function slowly varying at infinity.

Note that under our approach one of the key facts to prove Theorems 1, 2, 3 is convergence in distribution, as $n \rightarrow \infty$, of the products $\prod_{i=0}^{n} M_{i} \rho_{i}^{-1}$ of random matrices to a limit matrix whose distribution is not concentrated at zero matrix. It is known $(\underline{8})$ that for $p=2$ the products $\prod_{i=0}^{n} M_{i} \rho_{i}^{-1}$ of the positive bounded independent identically distributed $2 \times 2$ matrices $A_{i}=M_{i} \rho_{i}^{-1}$ converges in distribution, as $n \rightarrow \infty$, to a limit matrix whose distribution is not concentrated at zero matrix if and only if all the matrices $A_{i}$ have a common positive right or left eigenvector. Hence, for the 2-type process $Z(n)$ our assumption on existence of a common positive left eigenvector of the matrices $M$ is essential indeed.

Observe also that Assumption A1 covers non-degenerate random walks with zero mean and finite variance of there increments, as well as all non-degenerate symmetric random walks. In these cases $a=1 / 2$. Another example when Assumption A1 is valid gives the random walk, whose increments have distribution belonging to the domain of attraction of a stable law.

In conclusion we give an example where Assumption A2 is fulfilled (given that Assumption A0 is valid as well). Clearly, if the measure $\mathbf{F}$ generating our random environment has a bounded support, i.e., if there exists a $p$-dimensional cube $B=[0, b]^{p}, b>0$, such that $\mathbf{P}(\mathbf{F}(B)=1)=1$, then Assumption A2 holds since $\kappa(d)=0 \quad \mathbf{P}$-a.s. for $d>b$.

One can show that if $\mathbf{F}$ satisfies Assumption A0 and

$$
F(s)=\overline{1}-\frac{M(\overline{1}-s)}{1+\gamma(\overline{1}-s)}, s \in J^{p},
$$

where the $p$-dimensional random row vector $\gamma$ with positive components and the random matrix $M$ are such that the components of the vector $y=(M \overline{1}) /|\gamma|$ are uniformly bounded from below then Assumption A2 holds true.

Note that if the distribution of $X=\ln \rho$ has a regular varying tail then Assumptions A1 and A2 can be replaced by the following hypotheses (see (13) or (1)):

Assumption A1'. There exist constants $c_{n}, n \geq 0$, such that as $n \rightarrow \infty$ the scaled sums $c_{n} S_{n}$ converge weakly to a stable distribution $\mu$ with parameter $\beta \in(0,2]$. The limit law $\mu$ is not one-side, i.e., $0<$ $\mu\left(\mathbb{R}^{+}\right)<1$.

Assumption A2'. There exist $\varepsilon>0$ and $d \in \mathbf{N}_{0}$ such that

$$
\mathbf{E}\left(\ln ^{+} \kappa(d)\right)^{\beta+\varepsilon}<\infty,
$$

where $\beta$ is from Assumption A1'.

Note that Assumption A1' implies the validity of Assumption A1 with $a=\mu\left(\mathbb{R}^{+}\right)$, and Assumption A2 is stronger than Assumption A2' since $a \beta \leq 1$.

Corollary 1 Assume Assumptions AO and Al'. Then the statement of Theorem 2 remains true.

Using the proof of Theorem 3 and results of paper (1), one can obtain also the following statement.

Theorem 4 Assume Assumptions A0, A1', and A2'. Then the statement of Theorem 3 remains true.

\section{References}

[1] Afanasyev V.I., Geiger J., Kersting G., and Vatutin V.A. Criticality for branching processes in random environment. Ann. Prob., 33(2005), 2, p. 645-673.

[2] Athreya K.B. and Karlin S. On branching processes with random environments: I,II. Ann. Math. Statist., 42 (1971), 5, p. 1499-1520; 42(1971), 6, p. 1843-1858. 
[3] Bertoin J. and Doney R.A. On conditioning a random walk to stay nonnegtive. Ann. Prob., 22 (1994), p. 2152-2167.

[4] Dyakonova E.E. Asymptotic behavior of the probability of non-extinction for a multitype branching process in random environment. Discrete Math. Appl., 9 (1999), 2, p. 119-136.

[5] Dyakonova E.E., Geiger J., and Vatutin V.A. On the survival probability and a functional limit theorem for branching processes in random environment. Markov Process. Related Fields, 10 (2004), 2, p. 289-306.

[6] Geiger J. and Kersting G. The survival probability of a critical branching process in random environment. Theory Probab. Appl., 45(2000), 3, p. 607-615.

[7] Kaplan N. Some results about multidimentional branching processes with random environments. Ann. Prob., 2 (1974), 3, p. 441-455.

[8] Kesten H. and Spitzer F. Convergence in distribution of products of random matrices. Z. Wahrsch. verw. Geb., 67 (1984), 4, p. 363-386.

[9] Kozlov M.V. On the asymptotic behavior of the probability of non-extinction for critical branching processes in a random environment. Theory Probab. Appl., 21 (1976), 4, p. 791-804.

[10] Smith W.L. and Wilkinson W. On branching processes in random environment. Ann. Math. Statist., 40 (1969), 3, p. 814-827.

[11] Tanny D. On multitype branching processes in a random environment. Adv. Appl. Prob., 13 (1981), 3, p. 464-497.

[12] Vatutin V.A. and Dyakonova E.E. Galton-Watson branching processes in random environments, I: limit theorems. Theory Probab. Appl., 48(2003), 2, p. 314-336.

[13] Vatutin V.A. and Dyakonova E.E. Galton-Watson branching processes in random environments, II: joint distributions. Theory Probab. Appl., 49 (2004), 2, p. 275-308.

[14] Vatutin V.A. and Dyakonova E.E. Branching processes in random environment and bottlenecks in the evolution of populations. Theory Probab. Appl., 51(2006), 1. 
